\title{
The Effects of the Intensity of Cigarette Use and Antiretroviral Therapy on the Blood Lipid Profile of an Irish HIV Person
}

\author{
Charlie Slowey ${ }^{\mathrm{a}}$, Gabriella Rizzo ${ }^{\mathrm{a}}$
}

\begin{abstract}
Context: There are numerous studies on the effects of smoking and antiretroviral use separately on a HIV patient's blood lipid profile, however there has never been a study conducted that measures the intensity of cigarette use combined with antiretroviral therapy on a patient's blood lipid profile. Aims: To assess the effects of the intensity of cigarette usage and antiretroviral therapy on a patient's blood lipid profile. Setting and Design: This cross-sectional study was carried out in a regional HIV clinic in Cork University Hospital between June 2016 and August 2016. Methods: 40 patients were interviewed in order to gather cigarette usage data and patient files were reviewed in order to gather blood lipid profile results and antiretroviral therapy data. Results: There was no statistically significant difference $(\mathrm{P}>0.05)$ in the means of cholesterol (4.99 vs 5.47), high-density lipoprotein (1.22 vs 1.07$)$, low-density lipoprotein (3.13 vs 3.03), nor triglycerides (1.54 vs 2.45) in smokers versus non-smokers. Low-density lipoprotein in mild and moderate smokers was increased compared to non-smokers. Conclusions: These results show that smoking combined with nucleoside reverse transcriptase inhibitor use has no significant change in blood lipid measurements when compared to controls.
\end{abstract}

Keywords: Lipid, cholesterol, high density lipoprotein, low density lipoprotein, triglyceride, HIV, AIDS, human immunodeficiency virus, antiretroviral, anti-retroviral, antiretroviral drug, smoking, cigarette, tobacco.

\section{Background and Literature Review}

HIV infection and AIDS disease are increasingly becoming a problem of old age ${ }^{(1)}$ especially in the first world due to increased efficacy of antiretroviral drugs, multicombination drugs and a better understanding of the disease process $^{(2)}$. With HIV patients living longer and fuller lives more disease processes previously unconsidered must now be actively managed. It is well documented that cigarette smoking is a known risk factor for peripheral coronary and cerebrovascular vascular diseases ${ }^{(3)}$. It is estimated that nearly $20 \%$ of all coronary heart disease deaths can be attributed to smoking ${ }^{(4)}$. Research suggests that cigarette smoking produces a more atherogenic lipid profile characterized by higher total cholesterol levels along with higher triglyceride levels and lower levels of high density lipoprotein levels ${ }^{(5-7)}$. Smoking intensity is often correlated with a statistically significant increase in low-density lipoprotein ${ }^{(6,}{ }^{7}$. Highly active antiretroviral therapy is well known to cause dyslipidemia, with the incidence of between 15 and $30 \%{ }^{(8-12)}$, hypertriglyceridemia is the most common metabolic effect, followed by increased values of low-density protein, total cholesterol and low high-density lipoprotein levels ${ }^{(8,13,14)}$. This authors previous literature review sought to tie together the effects of smoking and antiretroviral use on a patient's blood lipid profile. The methods of that review and a summary of the results are included below.

\section{Review Methods:}

Step 1:

A systematic search of PubMed archives was carried out between 01/11/15 - 05/11/15. The review's search criteria are included below:

Anti-retroviral therapy search: Advanced search was used. In search box 1 the value 'Title/Abstract' was selected and the term 'Anti-retroviral therapy' was included. In search box 2 the AND value was selected as well as 'Title/Abstract' and the term 'lipid' was included. Full search equation: anti-retroviral therapy[Title/Abstract] AND lipid[Title/Abstract]. The search returned 36 results.

Cigarette Use search: Advanced search was used. In search box 1 the value 'Title' was selected and the term 'Smoking' was included. In search box 2 the value AND was used as well as the value 'Title' selected. The term 'Lipid' was included. Full search equation: Smoking[Title] AND Lipid[Title]. The search returned 153 results.

Combined Anti-retroviral therapy and Cigarette Use search: In the regular search box the terms 'smoking', 'antiretroviral' and 'lipid' were included. Full search equation: ("smoking"[MeSH Terms] OR "smoking"[All Fields]) AND "antiretroviral"[All Fields] AND ("lipids"[MeSH Terms] OR "lipids"[All Fields] OR "lipid"[All Fields]). Search returned 97 results.

The sum of the review's searches returned 286 articles and the following exclusion criteria were applied:

1. The article must be a clinical trial

2. The full text must be available

3. The article must not be in a different language

4. The trial must be carried out on homo sapiens

These criteria excluded 227 articles.

Step 2:

The abstracts of the remaining 59 articles were reviewed and 40 articles were excluded. The main reason for the exclusion was because the articles did not include relevent lipid profile data in their abstracts. 19 articles progressed to Step 3.

Step 3:

The author retrieved the full research for the remaining 19 abstracts and these articles were reviewed in depth and critiqued using the EBL Critical Appraisal Checklist. 


\section{Review Results:}

The previous review sought to quantify the effect that smoking has on blood lipid profiles, highly active antiretroviral therapy has on blood lipid profiles, and sought to compare these values to previous literature done on a combination of smoking and highly active antiretroviral therapy on blood lipid profits. The results generated for the cigarette use arm of this review were perhaps unsurprising with a mean increase in cholesterol $40 \%$, and mean increasing triglycerides of $80 \%$, a mean decrease in high-density level protein of $20 \%$, and the mean increase in low-density lipoprotein of $46 \%$.

The results from the highly active antiretroviral arm of the review suggested that cholesterol had a mean increase of $16 \%$, high-density level protein had a mean increase of $10 \%$, lowdensity lipoprotein an increase of $15 \%$ and triglycerides showed a non-significant increase of $5 \%$. What is interesting to note from this arm of the review is that of the eight studies reviewed only one had data for nucleoside reverse transcriptase inhibitor usage effects on blood lipid profiles.

The third arm of this review sought to compare the effects of the previous two rounds versus studies which showed the combined effects of smoking and highly active antiretroviral use on blood lipid profiles. Of note, the five studies that were reviewed included no data for the amount or intensity of cigarettes smoked. The results showed that total cholesterol was increased by a mean of $12 \%$, triglycerides were increased by mean of $75 \%$, and there was not enough data to support a mean value for high-density lipoprotein or low-density lipoprotein.

The authors concluded that while there was data for combined usage of smoking and antiretroviral therapy there was no data on the intensity of cigarette usage and how that effects a patient's blood lipid profile. Of the limited data that was available it was shown that smoking and antiretroviral therapy cause a more atherogenic blood lipid profile however they do not have an additive deleterious effect on patient's blood lipid profile.

\section{Aims and Objectives}

Aim: To evaluate the effects of smoking over a range of pack year levels with the most common antiretroviral therapy treatments in Cork University Hospital on the blood lipid profile of HIV infected patients.

Objectives: Interview a wide range of patients with respect to age, sex, and smoking pack year value. Obtain comprehensive antiretroviral therapy data on each patient. Obtain up-to-date lipid profile values for every patient. Compare blood lipid values for smokers versus non-smokers and in the smoking category compare the range of pack year values with blood lipid profile data given a patient's antiretroviral therapy regimen.

\footnotetext{
Methods

Study design: This cross-sectional study was carried out between June and August in the weekly HIV clinic in Cork University Hospital. 50 participants were randomly selected over a number of weeks from the Cork University Hospital HIV clinic and were subsequently interviewed on the day of selection. Major inclusion criteria included; age between 18 and 65 years old, previous diagnosis of HIV infection, and a lab cholesterol value no more than six months old. Exclusion criteria included any participant with a previous history of
}

cardiovascular disease (e.g. myocardial infarction), any acute injury or infectious episode over the last six weeks any antibiotic/antimicrobial therapy over the last six weeks, and any family history of lipid disorders. A further 10 patients were excluded from the study due to a lack of any fasting cholesterol value. Non-smokers with a previous diagnosis of HIV were recruited as matched controls for the participants in the study. Study measures: A number of parameters were collected during the interview including age smoking status pack your status, healthy versus non-healthy diet, and the participants exercise levels (light, moderate, heavy). See Appendix 3, 4, 5 \& 6 for invitation letter, participant information sheet, full interview questionnaire and consent form. Following a chart review, further parameters were collected including, type of drug currently prescribed, the length of time the patient was on therapy, the CD4 plus count, and the HIV RNA titer. Following this interview, the patient's blood lipid results were also recorded. These results were within six months of the interview and included cholesterol level, high-density lipoprotein level, triglyceride level, and a calculated low-density lipoprotein level calculated by the Friedewald formula ${ }^{(15)}$. Participants were separated based on their pack year status (using formula (number of cigarettes smoked per day multiplied symbol number of years smoked) divided by 20) from non-smoker to low, moderate and high smoking levels. Blood was drawn after 12 hours fasting with staple food for two days. Enzymatic method was used to estimate total cholesterol and triglycerides. HDL was determined by precipitation. LDL cholesterol was then calculated using the Friedewald (Formula: $\mathrm{LDL}=\mathrm{TC}-$ HDL - TG/2.17 (mmol/L).

Ethics: This study obtained ethical approval from the Cork Research Ethics Committee. See Appendix 1 for full ethical approval.

Timetable: The authors collected data over three months in 2016, June - August, with data analysis and article editing taking place in September. See Appendix 2 for full timeline.

Data Analysis: Study parameters were recorded in SPSS software (IBM SPSS Statistics 2016) for all participants along with identifying information being stored on an encrypted Excel file on an encrypted USB stick in the primary investigators office. Data was analyzed by SPSS software using mean comparisons and Pearson correlations. Graphing results was performed by PRISM GraphPad and IBM SPSS Statistics 2016.

\section{Results:}

Of the 40 participants included in the final data analysis $30(80 \%)$ were male. The mean age was 41.9 years (standard deviation of 8.09) and a range of 23 to 58 years. $97.5 \%$ of participants were prescribed a nucleoside reverse transcriptase inhibitor drug combination with $90 \%$ on the combination tenofovir/emtricitabine. Study participants were further classified into their nucleoside reverse transcriptase inhibitor therapy plus additional antiretroviral therapy. Participants were stratified into smokers and non-smokers and the average means of each; cholesterol, high-density lipoprotein, low-density lipoprotein, and triglycerides were compared. Smokers were further classified into mild smokers (.1 to 15 pack year), moderate (15.1 to 30 pack year), and heavy (greater than 30 pack year) for further analysis. All 40 research participants had cholesterol values, 26 had high-density lipoprotein values and low-density lipoprotein values, and 29 had triglyceride values. 
The mean values for cholesterol, high-density lipoprotein, lowdensity lipoprotein, and triglycerides in smokers versus nonsmokers is shown in Table 1.

Table l: A comparison of Smoking Status versus Cholesterol, High Density Lipoprotein, Low Density Lipoprotein, and Triglycerides.

\begin{tabular}{|c|c|c|c|c|c|}
\hline \multicolumn{2}{|c|}{ Sm oking Status } & Chol ester ol & $\begin{array}{l}\text { High Density } \\
\text { Lipoprotein }\end{array}$ & $\begin{array}{l}\text { Low Density } \\
\text { Lipoprotein }\end{array}$ & Triglycerides \\
\hline & $\begin{array}{l}\text { Mean } \\
\text { Participants } \\
\text { Standard deviation }\end{array}$ & $\begin{array}{l}4.9870 \\
23 \\
90167\end{array}$ & $\begin{array}{l}1.2287 \\
15 \\
28804\end{array}$ & $\begin{array}{l}3.1333 \\
15 \\
74706\end{array}$ & $\begin{array}{l}1.5406 \\
16 \\
95847\end{array}$ \\
\hline No & $\begin{array}{l}\text { Mean } \\
\text { Participants } \\
\text { Standard deviation }\end{array}$ & $\begin{array}{l}5.4706 \\
17 \\
1.39855\end{array}$ & $\begin{array}{l}1.0727 \\
11 \\
21597\end{array}$ & $\begin{array}{l}3.0391 \\
11 \\
46798\end{array}$ & $\begin{array}{l}2.4500 \\
13 \\
1.77310\end{array}$ \\
\hline
\end{tabular}

Cholesterol values had an average of $4.99 \mathrm{mmol} / \mathrm{l}$ versus $5.474 \mathrm{mmol} / \mathrm{l}$ for non-smokers, high-density level protein had a value of $1.28 \mathrm{mmol} / \mathrm{l}$ for smokers versus $1.074 \mathrm{mmol} / \mathrm{l}$ for non-smokers, low-density lipoprotein had a value of 3.13 $\mathrm{mmol} / \mathrm{l}$ for smokers versus $3.044 \mathrm{mmol} / \mathrm{l}$ for non-smokers, triglycerides had a value of $1.544 \mathrm{mmol} / \mathrm{l}$ for smokers versus $2.474 \mathrm{mmol} / \mathrm{l}$ for non-smokers. The mean values for cholesterol, high-density lipoprotein, low-density lipoprotein, and triglycerides versus the intensity of smoking followed a similar trend to the previous values in that participants who smoked had lower cholesterol, higher high density lipoprotein, and lower triglycerides. However, when smoking intensity was stratified smokers showed a higher low-density lipoprotein mean. These results are shown in more detail in Table $\mathbf{2}$ and Figure 1.

Table 2: A comparison of means of Heavy, Moderate, Mild and No use of Cigarettes versus Blood Lipid

Profile levels.

\begin{tabular}{|c|c|c|c|c|c|}
\hline \multicolumn{2}{|c|}{ Cigarette Use } & \multirow{4}{*}{$\begin{array}{r}\text { Cholesterol } \\
4.4333 \\
3 \\
.70238 \\
\end{array}$} & \multirow{4}{*}{$\begin{array}{l}\text { High Density } \\
\text { Lipoprotein } \\
9800 \\
1\end{array}$} & \multirow{4}{*}{$\begin{array}{l}\text { Low Density } \\
\text { Lipoprotein } \\
1.9000 \\
1\end{array}$} & \multirow{4}{*}{$\begin{array}{r}\text { Triglycerides } \\
1.8600 \\
1\end{array}$} \\
\hline Heavy Use & Mean & & & & \\
\hline & Participants & & & & \\
\hline & Standard Deviation & & & & \\
\hline Moderate & Mean & 4.9778 & 1.3520 & 3.4000 & 1.5833 \\
\hline \multirow[t]{2}{*}{ Use } & Participants & 9 & 5 & 5 & 6 \\
\hline & Standard Deviation & .84377 & 31650 & .41231 & 1.39415 \\
\hline \multirow[t]{3}{*}{ Mild Use } & Mean & 5.1455 & 1.1878 & 3.1222 & 1.4767 \\
\hline & Participants & 11 & 9 & 9 & 9 \\
\hline & Standard Deviation & 1.00036 & .27567 & .81052 & 69929 \\
\hline Non- & Mean & 5.4706 & 1.0727 & 3.0391 & 2.4500 \\
\hline \multirow[t]{2}{*}{ Smoker } & Participants & 17 & 11 & 11 & 13 \\
\hline & Standard Deviation & 1.39855 & .21597 & .46798 & 1.77310 \\
\hline
\end{tabular}




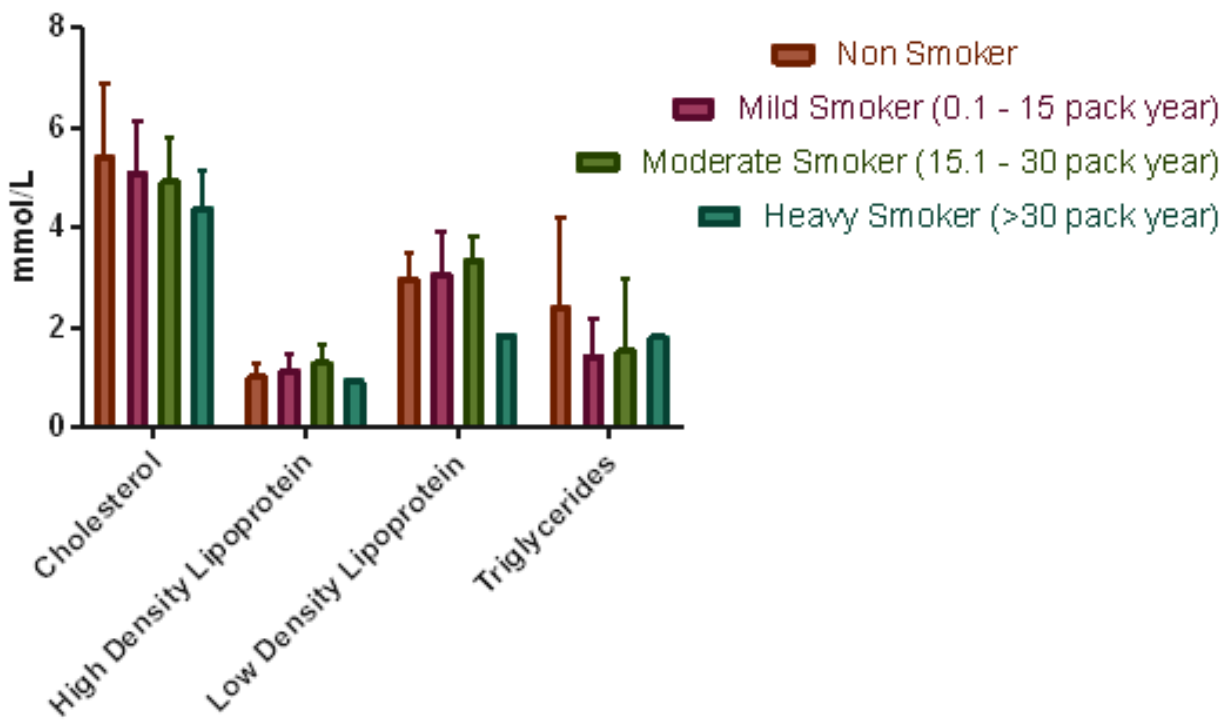

Blood Lipid Profile Components

Figure 1: A comparison of means of Non-Smokers vs. Mild, Moderate, and Heavy Cigarette Use.

Pearson correlations showed no statistically significant correlation between pack year smoking level and cholesterol, high-density lipoprotein, low-density lipoprotein, and triglycerides. All values were within the -0.3 to 0.3 range. Details of these correlations are detailed in Table 3.

Table 3: Pearson Correlations betw een Pack Year values and Cholesterol, High Density Lipoprotein, Low Density

Lipoprotein, and Triglycerides.

\begin{tabular}{|c|c|c|c|c|c|}
\hline & & Chol esterol & $\begin{array}{l}\text { High Density } \\
\text { Lipoprotein }\end{array}$ & $\begin{array}{l}\text { Low Density } \\
\text { Lipoprotein }\end{array}$ & Triglycerides \\
\hline \multirow{3}{*}{$\begin{array}{l}\text { Pack Year } \\
\text { value }\end{array}$} & Pearson Correlation & -.258 & .291 & -.013 & -.222 \\
\hline & Significance ( 2 tailed) & .109 & .150 & .952 & .248 \\
\hline & Participants & 40 & 26 & 25 & 29 \\
\hline
\end{tabular}

Independent samples t-test for each cholesterol, highdensity like protein, low-density lipoprotein, and triglycerides in smokers versus non-smokers all showed a non-statistically significant difference in means: a mean decrease of 0.48 $\mathrm{mmol} / \mathrm{l}$ in cholesterol values for smokers, mean increase of $0.16 \mathrm{mmol} / \mathrm{l}$ in high-density lipoprotein values for smokers, and mean increase of $0.1 \mathrm{mmol} / \mathrm{l}$ low-density lipoprotein values for smokers, and the mean decrease of $0.91 \mathrm{mmol} / \mathrm{l}$ in triglyceride values for smokers. The interplay between different antiretroviral drug regimens for smokers verses nonsmokers is detailed in Figure 2. Nucleoside reverse transcriptase inhibitor plus protease inhibitor showed a mean cholesterol value $4.2 \mathrm{mmol} / \mathrm{l}$ versus $5.2 \mathrm{mmol} / \mathrm{l}$ for smoking verses non-smoking. Nucleoside reverse transcriptase inhibitor plus integrase inhibitor showed a mean cholesterol of 5.3 $\mathrm{mmol} / \mathrm{l}$ versus $5.3 \mathrm{mmol} / \mathrm{l}$ for smoking verses non-smoking. Nucleoside reverse transcriptase inhibitor plus non-nucleoside reverse transcriptase inhibitors showed a mean cholesterol 5.2 $\mathrm{mmol} / \mathrm{l}$ versus $4.8 \mathrm{mmol} / \mathrm{l}$ for smokers versus non-smokers. In the other category any study participant who was not prescribed the previous three drug combination regimes was classed into this category, the mean cholesterol values for this category were $5 \mathrm{mmol} / \mathrm{l}$ versus $7.5 \mathrm{mmol} / \mathrm{l}$ for smokers versus non-smokers. All of these results were statistically nonsignificant. In terms of the effects of anti-retroviral therapy on a person's CD4+ count, there was a clear statistically significant positive correlation $(0.402, \mathrm{P}=0.01)$ between years spent on anti-retroviral therapy and CD4+ count. 


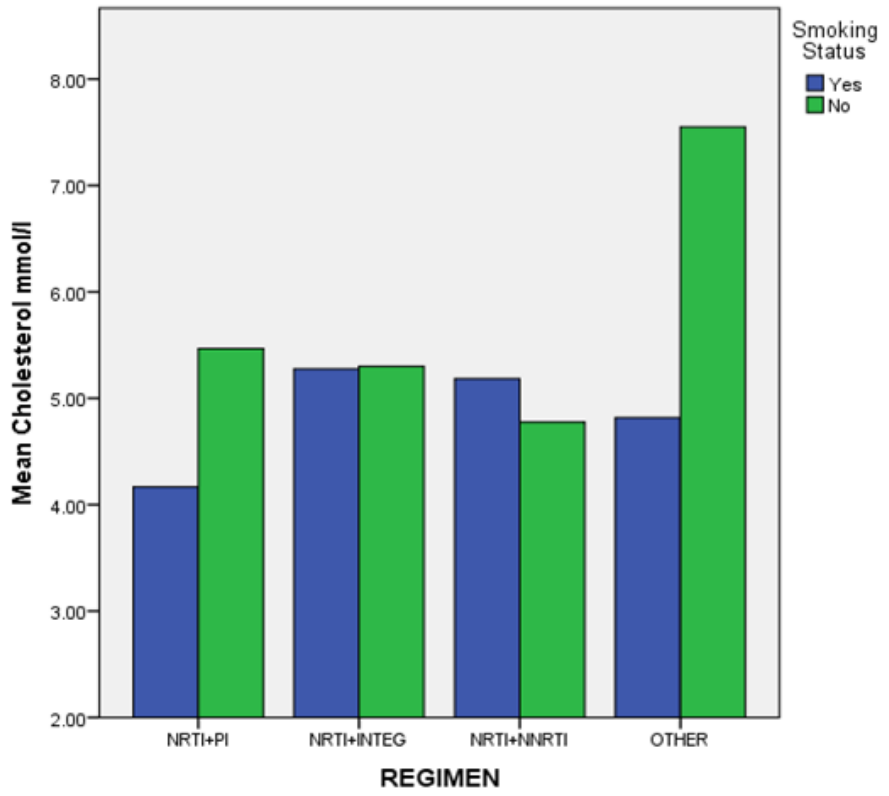

Figure 2: Mean Cholesterol Levels vs Antiviral Regimen

NRTI+PI: Nucleoside reverse transcriptase inhibitor plus protease inhibitor. NRTI+INTEG: nucleoside reverse transcriptase inhibitor plus integrase inhibitor. NTRI+NNRTI: Nucleoside reverse transcriptase inhibitor plus non-nucleoside reverse transcriptase inhibitor. OTHER: Any other regimen.

\section{Discussion}

It is well known that smoking has a negative impact on blood lipid profile cholesterol high-density level protein, lowdensity lipoprotein, and triglycerides ${ }^{(16)}$. The specific abnormalities include an increase in cholesterol levels, lowdensity lipoprotein levels, and triglycerides. High density lipoprotein, a marker of 'good' cholesterol, is decreased by smoking ${ }^{(17)}$. What is interesting to note is that most data on the effects of antiretroviral drugs on blood lipid profiles included only protease inhibitors and non-nucleoside reverse transcriptase inhibitors ${ }^{(9,10,14,18,19)}$. This study focuses mostly on nucleoside reverse transcriptase inhibitors as a base to antiretroviral therapy. After conducting a literature review to confirm this, it was concluded that this a one-of-a-kind study on the intensity of cigarette smoking and use of nucleoside reverse transcriptase inhibitor as a base drug on blood lipid profiles. The results generated in the study would indicate that smoking combined with nucleoside reverse transcription inhibitors as a base drug have a broadly positive effect on a patient's blood lipid profile measurements. This is seen with cholesterol and triglyceride mean levels lower in smokers than non-smokers. High density lipoprotein is raised in mild and moderate smokers as compared to non-smokers. Low Density Lipoprotein change is the only indication that smoking combined with nucleoside reverse transcriptase inhibitors has an adverse effect on a patient's blood lipid profile. The values for heavy smokers in the high-density lipoprotein, low-density lipoprotein, and triglyceride categories must be discounted as there was only one participant who had available values for each of these categories. Thus, for the heavy smoking category, the values for high-density lipoprotein, low-density lipoprotein, and triglycerides cannot be used as a comparison to mild and moderate users. An analysis of the data using Pearson correlations showed no statistically significant correlations between cholesterol, high-density lipoprotein, low-density lipoprotein, and triglycerides with pack year levels. This again goes against the research previously published on this topic and suggests that nucleoside reverse transcriptase inhibitors produce a favorable change to a patient's blood lipid profile. Due to the lack of statistically significant correlations it is clear to the authors that more participants are needed in order to delineate the association between blood lipid profile levels and intensity of cigarette use in HIV patients who are prescribed nucleoside reverse transcriptase inhibitor therapy in combination on other drugs. In the final figure (Figure 2), that compares antiretroviral regimens versus smokers and non-smokers it is clear that there is no great difference between the regimens as a whole and between smokers and non-smokers in each regimen. The biggest difference seen here is in the other category where nonsmokers had a much higher cholesterol value than smokers. This can be attributed to low study numbers and diversity of antiretroviral therapy within this group. The final result of this study, the positive correlation between years on therapy and CD4+ count, is perhaps the most heartening as it shows that anti-retroviral therapy does work. This is due to the predictive nature of higher CD4+ counts on increased mortality rates among HIV positive patients ${ }^{(20)}$

\section{Strengths of Project:}

The strengths of this research project follow from its location. As the project was located in a tertiary care center, access to patients was quick and easy and the number of potential patients was large. Furthermore, due to the outpatient nature of the clinics patient follow-up was regular, scheduled, and easy to track. Advice from current physicians working in the outpatient clinic allow easy identification of patients who would be willing to talk about their condition in this very 
stigmatized area. The interview conducted with the patients was also a strength in the fact that it was quick, easy to follow, and easy to answer. Furthermore, having a private room to conduct these interviews in assured the patients their privacy and allowed the doctor-patient relationship to work to its fullest extent to glean answers. The collection of drug regimen data and blood lipid profile data was also of great ease as the patient files were located in the outpatient department during time of interview and the blood lipid profile results were located on the local hospital intranet which was easily accessed after the day's interviews. The cost associated with this project must also be counted as another strength, total costs for this project came to under $€ 20$ due to the fact that, by and large, the only costs associated with this were for printing the patient information sheet and consent forms.

\section{Weaknesses of Project:}

The main weakness of this project is apparent from the data set. Half of the research subjects did not have any data on the blood lipid profile values of high-density lipoprotein, lowdensity lipoprotein, nor triglycerides. As seen from the results generated above this makes data analysis difficult and nongeneralizable. It is due to this key weakness that the authors were unable to find a correlation between smoking HIV drug regimen and lipid profile values. After consultation with the physicians in the outpatient department it was clear blood lipid profile testing was not a standard investigation for this outpatient department. Because of this the authors had to order a blood lipid profile test on second patient follow-up appointment. This, obviously, was associated with poor follow-up due to patients not attending for follow-up appointment or a lack of resources in the outpatient department on the follow-up appointment date (with patient refusal to see phlebotomist as the primary reason for failure). The time constraints for this project were also considerable due to the limited time available for data collection. As data collection had to occur over the summer months during one day a week, it was difficult to recruit patients to the study. Along the lines of patient recruitment, another weakness of this study was also very apparent. As mentioned in the strengths above the authors had great help from the physicians of the outpatient department in selecting suitable patients. However, it also highlighted a problem with patient selection; namely that one in 10 patients were willing to talk to the primary investigator. This can be summarized in one word and that word is stigma. The stigma of HIV disease is very apparent today, due to this it was hard to convince sufferers to talk about their disease with a student. Added to this is the nature of the outpatient department; it was a shared clinic with infectious diseases and rheumatology patients sitting together. Requesting patient compliance with this study in a private manner was challenging for both investigators and patients. If this study were to have been conducted by a physician of infectious diseases in the outpatient department as an adjunct to a patient follow-up appointment, the proportion of patient recruitment would have significantly increased. The subjective nature of the diet and exercise questions on the patients question sheet was also another weakness. This relied on patient's long-term recall of exercise habits and dietary habits, which of course is subject to errors of recall. Because of this it is difficult to measure the effect that these variables had on a patient's blood lipid profile. Furthermore, this project would have benefited from several data collectors due to the high turnover of HIV clinic. Investigators found that suitable patients were being missed due to those patients having already conducted their follow-up appointment before the investigators were finished with a previous patient. As mentioned above, when the compliance rate was one in 10 patients, this led to a greatly decreased number of participants in the study.

\section{References:}

1. Helleberg M, May MT, Ingle SM, Dabis F, Reiss P, Fatkenheuer G, et al. Smoking and life expectancy among HIV-infected individuals on antiretroviral therapy in Europe and North America. AIDS (London, England). 2015;29(2):221-9.

2. Maartens G, Celum C, Lewin SR. HIV infection: epidemiology, pathogenesis, treatment, and prevention. The Lancet.384(9939):258-71.

3. Ockene IS, Miller NH. Cigarette Smoking, Cardiovascular Disease, and Stroke. A Statement for Healthcare Professionals From the American Heart Association. 1997;96(9):3243-7.

4. Doll R, Peto R, Boreham J, Sutherland I. Mortality in relation to smoking: 50 years' observations on male British doctors. Bmj. 2004;328(7455):1519.

5. Joshi N, Shah C, Mehta H, Gokhle P. Comparative study of lipid profile on healthy smoker and non smokers. Int J Med Sci Public Health. 2013;2(3):622-6.

6. Gamit KS, Nanavati MG, Gohel PM, R.N.Gonsai. EFFECTS OF SMOKING ON LIPIDS PROFILE. IJCRR. 2013;5(17):36-42.

7. Devaranavadgi B. B ABS, Kashinath R. T \& Hundakari I. A. Effect of Cigarette Smoking on Blood Lipids - A Study in Belgaum, Northern Karnataka, India. Global Journal of Medical Research. 2012;12(6):57 - 61.

8. Ngala RA, Fianko K. Dyslipidaemia and dysglycaemia in HIV-infected patients on highly active anti-retroviral therapy in Kumasi Metropolis. African health sciences. 2013;13(4):1107-16.

9. Molina JM, Andrade-Villanueva J, Echevarria J, Chetchotisakd P, Corral J, David N, et al. Oncedaily atazanavir/ritonavir compared with twicedaily lopinavir/ritonavir, each in combination with tenofovir and emtricitabine, for management of antiretroviral-naive HIV-1-infected patients: 96week efficacy and safety results of the CASTLE study. Journal of acquired immune deficiency syndromes (1999). 2010;53(3):323-32.

10. Lai S, Lai H, Celentano DD, Vlahov D, Ren S, Margolick J, et al. Factors associated with accelerated atherosclerosis in HIV-1-infected persons treated with protease inhibitors. AIDS patient care and STDs. 2003;17(5):211-9.

11. Duro M, Sarmento-Castro R, Almeida C, Medeiros $\mathrm{R}$, Rebelo I. Lipid profile changes by high activity anti-retroviral therapy. Clinical biochemistry. 2013;46(9):740-4.

12. Adewole OO, Eze S, Betiku Y, Anteyi E, Wada I, Ajuwon Z, et al. Lipid profile in HIV/AIDS patients 
in Nigeria. African health sciences. 2010;10(2):1449 .

13. Daniyam C, Iroezindu M. Lipid Profile of AntiRetroviral Treatment-Naive HIV-Infected Patients in Jos, Nigeria. Annals of medical and health sciences research. 2013;3(1):26-30.

14. Ete T, Ranabir S, Thongam N, Ningthoujam B, Rajkumar N, Thongam B. Metabolic abnormalities in human immunodeficiency virus patients with protease inhibitor-based therapy. Indian journal of sexually transmitted diseases. 2014;35(2):100-3.

15. Friedewald WT, Levy RI, Fredrickson DS. Estimation of the Concentration of Low-Density Lipoprotein Cholesterol in Plasma, Without Use of the Preparative Ultracentrifuge. Clinical Chemistry. 1972;18(6):499-502.

16. Gepner AD, Piper ME, Johnson HM, Fiore MC, Baker TB, Stein JH. Effects of smoking and smoking cessation on lipids and lipoproteins: outcomes from a randomized clinical trial. American heart journal. 2011;161(1):145-51.
17. Batic-Mujanovic O, Zildzic M, Beganlic A, Kusljugic Z. [The effect of cigarette smoking on HDL-cholesterol level]. Medicinski arhiv. 2006;60(6 Suppl 2):90-2.

18. Mittal A, Achappa B, Madi D, Chowta MN, Ramapuram JT, Rao S, et al. The development of metabolic risk factors after the initiation of the second line anti- retroviral therapy. Journal of clinical and diagnostic research : JCDR. 2013;7(2):265-8.

19. Asztalos BF, Schaefer EJ, Horvath KV, Cox CE, Skinner S, Gerrior J, et al. Protease inhibitor-based HAART, HDL, and CHD-risk in HIV-infected patients. Atherosclerosis. 2006;184(1):72-7.

20. Hoffmann CJ, Schomaker M, Fox MP, Mutevedzi P, Giddy J, Prozesky H, et al. CD4 count slope and mortality in HIV-infected patients on antiretroviral therapy: multicohort analysis from South Africa. Journal of acquired immune deficiency syndromes (1999). 2013;63(1):34-41. 